\title{
Azacitidine might be beneficial in a subgroup of older AML patients compared to intensive chemotherapy: a single centre retrospective study of 227 consecutive patients
}

Lieke H van der Helm ${ }^{1 \dagger}$, Ellen RM Scheepers ${ }^{1 \dagger}$, Nic JGM Veeger ${ }^{1}$, Simon MGJ Daenen ${ }^{1}$, André B Mulder ${ }^{2}$, Eva van den Berg ${ }^{3}$, Edo Vellenga ${ }^{{ }^{*}}$ and Gerwin Huls $^{1}$

\begin{abstract}
Background: Treatment options in older acute myeloid leukaemia (AML) patients include intensive chemotherapy, best supportive care (BSC), and hypomethylating agents. Currently, limited data is available on hypomethylating agents in older AML patients in unselected patient populations.

Methods: To compare the effectiveness of azacitidine with conventional therapy, we collected data of 227 consecutive AML patients ( $\geq 60$ years) who were treated with azacitidine $(N=26)$, intensive chemotherapy $(N=90)$, or BSC $(N=97)$.

Results: Azacitidine-treated patients were older and had more comorbidities, but lower white blood cell- and bone marrow blast counts compared with intensive chemotherapy patients. Complete or partial response was achieved in $42 \%$ of azacitidine-treated patients and in $73 \%$ of intensive chemotherapy patients $(P=0.005)$. However, the overall survival (OS) was similar (1-year-OS 57\% versus 56\%, $P=0.93 ; 2$-year-OS $35 \%$ versus $35 \%, P=0.92$ ), and remained similar after correction for risk factors in a multivariate analysis. Patients treated with BSC had an inferior OS (1-yearand 2-year-OS 16\% and 2\%, $P<0.001$ ). Compared to intensive chemotherapy, azacitidine-treated patients spent less days in the hospital (median in first three months 0.5 versus $56, P<0.001$ ), and needed less red blood cell and platelet transfusions (median per month 2.7 versus $7, P<0.001$ and 0.3 versus $5, P<0.001$ ) in the first three months.
\end{abstract}

Conclusions: Azacitidine treatment is associated with a comparable OS but higher tolerability in a subgroup of older AML patients compared with intensive chemotherapy. Patients receiving BSC had a poor prognosis.

Keywords: Acute myeloid leukaemia, Older age, Azacitidine, Intensive chemotherapy, Best supportive care

\section{Background}

Acute myeloid leukaemia (AML) is characterised by a differentiation defect of haematopoietic stem- and progenitor cells, leading to the accumulation of blast cells and cytopenias. The incidence of AML increases with age, with a median age at diagnosis of approximately 70 years [1]. Older AML patients generally have a poor prognosis compared to younger patients due to a higher incidence of

\footnotetext{
* Correspondence: e.vellenga@umcg.nl

${ }^{\dagger}$ Equal contributors

'Department of Haematology, University Medical Centre Groningen,

University of Groningen, Hanzeplein 1, Groningen 9713 GZ, The Netherlands Full list of author information is available at the end of the article
}

comorbidities, higher rates of treatment related mortality, and adverse disease characteristics, associated with resistant disease and relapses [2-5]. Median overall survival (OS) of patients over 60 years of age treated with intensive chemotherapy is less than 1 year, with complete remission (CR) rates of about $50 \%$ and a treatment related mortality of at least $15 \%$, indicating an unfavourable risk-benefit ratio of intensive chemotherapy [6,7].

Nevertheless, several studies suggest that older AML patients benefit from treatment. A small randomized clinical trial by the HOVON study group and an analysis of the Swedish Acute Leukemia Registry showed that standard intensive treatment improves early death

\section{() Biomed Central}


rates and long-term survival compared to best supportive care only (BSC) in older patients [8-10]. In an additional prospective randomized trial it was demonstrated that lowdose cytarabine treatment was superior to BSC and hydroxyurea in patients with favourable- or intermediate-risk cytogenetics [11].

The optimal treatment of older AML patients in daily clinical practice remains challenging. A choice should be made between intensive chemotherapy, less intensive treatment, and palliation, considering individual risks and benefits $[7,12]$. To guide physicians in their decisions, several prognostic factors have been identified and risk scores have been developed based on age, performance status, comorbidities, cytogenetics, molecular markers, clinical variables, and laboratory measurements [13-17].

Recently, the DNA methyltransferase inhibitor azacitidine has become available for MDS and AML patients with up to $30 \%$ bone marrow blast. A superior OS has been demonstrated in AML patients with $20-30 \%$ bone marrow blasts treated with azacitidine compared to conventional treatment $[18,19]$. Two recent studies showed a beneficial outcome in previously untreated AML patients, including patients with more than $30 \%$ bone marrow blasts, who were treated with azacitidine [20,21]. However, limited data is available on the treatment of older unselected AML patients with azacitidine compared to conventional treatment options. To study the impact of azacitidine and conventional care options in routine clinical practice, we analysed the treatment results of 227 consecutive newly diagnosed AML patients of 60 years and older in our centre.

\section{Results}

\section{Baseline characteristics of the study population}

The study population included 227 consecutive newly diagnosed AML patients of 60 years and older. Patients were treated according to one of the following main strategies: 26 (11\%) patients were treated with azacitidine, 90 (40\%) patients were treated with intensive chemotherapy, 97 (43\%) patients were treated with best supportive care (BSC), and $14(6 \%)$ patients were diagnosed with acute promyelocytic leukaemia (APL) and treated with all-trans retinoic acid (ATRA)-containing intensive chemotherapy (Additional file 1: Figure S1). These fourteen APL patients were excluded from comparisons of azacitidine with intensive chemotherapy, because of the alternative treatment strategy and superior OS. Patients who were treated with azacitidine completed a median of $6(1-30)$ cycles. All azacitidine patients started with the standard dose (7 days $75 \mathrm{mg} / \mathrm{m}^{2} /$ day), except for one patient with pancytopenia and malaise at diagnosis who received $50 \mathrm{mg} / \mathrm{m}^{2} /$ day. A dose reduction of $30 \%$ was made in one patient after $10 \mathrm{cy}$ cles and a schedule change from 7 to 5 days was applied in one patient after 13 cycles. Failure to receive at least three cycles of azacitidine was reported in six (23\%) patients; the reason of interruption was early death $(N=4)$, or pancytopenia $(N=2)$.

Baseline patient- and disease characteristics of the different treatment groups are shown in Table 1. Considering patient related factors: patients who were treated with azacitidine and BSC were significantly older than patients treated with intensive chemotherapy $(P<0.001)$. Patients receiving azacitidine had a superior performance $(P=0.002)$, and patients receiving BSC had a worse performance $(P=0.003)$ compared to patients receiving intensive chemotherapy. The HCT-comorbidity score was worse in patients treated with azacitidine $(P=0.029)$ and BSC $(P<0.001)$ compared to intensive chemotherapy. Considering disease related factors: the percentage of secondary AML (including therapy related AML, prior MDS, and prior myeloproliferative neoplasms) was not significantly different between the treatment groups $(P=0.089)$. Patients who were treated with azacitidine had lower bone marrow $(\mathrm{BM})$ blast counts $(P<0.001)$, and white blood cell (WBC) counts $(P<0.001)$ compared to patients treated with intensive chemotherapy. Indeed, none of the patients treated with azacitidine had $\mathrm{WBC} \geq 15 \times 10^{9} / \mathrm{l}$. The cytogenetic risk score was not significantly different in patients treated with azacitidine compared with intensive chemotherapy $(P=0.48)$, but cytogenetic risk was worse in patients treated with BSC compared to intensive chemotherapy $(P=0.005)$. In 23 patients, the karyotype was not evaluated at baseline.

Allogeneic hematopoietic stem cell transplantation (allo-SCT) was applied in 14 patients following induction chemotherapy and also in one patient after azacitidine treatment. Baseline characteristics of these allo-SCT patients are shown in Additional file 2: Table S1. All alloSCT patients received reduced intensity conditioning with fludarabine (30 mg/m $\mathrm{m}^{2}$ for 3 subsequent days) and 2 Gray total body irradiation (TBI) before transplantation. All patients received mobilised peripheral blood stem cells, that were obtained from HLA matched siblings in 11 patients and from matched unrelated donors in 4 patients. Since transplant strategies have evolved during the study period, the last years, patients under the age of 70 in CR after two cycles of chemotherapy with a 10/10 matched donor available received an allogeneic hematopoietic cell transplantation. Indeed, of the patients younger than 70 years in CR, 12 (24\%) received an allo-SCT, which is $10(48 \%)$ considering this patient group since 2008 .

\section{Response}

Response (CR, PR) was achieved in 11 (42\%) patients who were treated with azacitidine, and in 66 (73\%) patients who were treated with intensive chemotherapy, which was significantly different $(P<0.001$; Table 2$)$. Of the 55 patients treated with 6-mercaptopurine, two patients met criteria 
Table 1 Baseline patient- and disease characteristics by treatment strategy

\begin{tabular}{|c|c|c|c|c|c|c|}
\hline & \multirow{2}{*}{$\begin{array}{l}\text { All patients } \\
(N=213)\end{array}$} & \multirow{2}{*}{$\begin{array}{l}\text { Azacitidine } \\
(N=26)\end{array}$} & \multirow{2}{*}{$\begin{array}{c}\text { Intensive } \\
\text { chemotherapy } \\
(N=90)\end{array}$} & \multirow[t]{2}{*}{ BSC $(N=97)$} & \multicolumn{2}{|c|}{$P$-value } \\
\hline & & & & & Overall & Aza vs IC \\
\hline \multicolumn{7}{|l|}{ Age } \\
\hline Median (range) & $68(60-96)$ & $70(60-81)$ & $66(60-74)$ & $71(60-96)$ & $<0.001$ & $<0.001$ \\
\hline$\geq 70$ years & $80(38 \%)$ & $14(54 \%)$ & $10(11 \%)$ & $56(58 \%)$ & $<0.001$ & $<0.001$ \\
\hline Sex & & & & & 0.48 & 0.27 \\
\hline Male & $119(56 \%)$ & $17(65 \%)$ & $47(52 \%)$ & $55(57 \%)$ & & \\
\hline Performance score & & & & & $<0.001$ & 0.002 \\
\hline$\geq 2$ & $121(59 \%)$ & $5(19 \%)$ & $47(54 \%)$ & $69(75 \%)$ & & \\
\hline HCT-comorbidity index & & & & & $<0.001$ & 0.029 \\
\hline Low $(0)$ & $98(46 \%)$ & $9(35 \%)$ & $57(63 \%)$ & $32(33 \%)$ & & \\
\hline Intermediate (1-2) & $66(31 \%)$ & $8(31 \%)$ & $18(20 \%)$ & $40(41 \%)$ & & \\
\hline $\operatorname{High}(>2)$ & $49(23 \%)$ & $9(35 \%)$ & $15(17 \%)$ & $25(26 \%)$ & & \\
\hline AML FAB classification & & & & & 0.27 & 0.28 \\
\hline Mo/M1 & $41(20 \%)$ & $4(17 \%)$ & $21(21 \%)$ & $16(19 \%)$ & & \\
\hline M2 & $87(42 \%)$ & $8(33 \%)$ & $40(40 \%)$ & $39(47 \%)$ & & \\
\hline M4/M5 & $51(25 \%)$ & $11(46 \%)$ & $22(22 \%)$ & $18(22 \%)$ & & \\
\hline M6/M7 & $13(6 \%)$ & $1(4 \%)$ & $4(4 \%)$ & $8(10 \%)$ & & \\
\hline AML type & & & & & 0.089 & 0.056 \\
\hline De novo & $139(65 \%)$ & $13(50 \%)$ & $65(72 \%)$ & $61(63 \%)$ & & \\
\hline Secondary & $74(35 \%)$ & $13(50 \%)$ & $25(28 \%)$ & $36(37 \%)$ & & \\
\hline \multicolumn{7}{|l|}{ Bone marrow blasts } \\
\hline Median (range) & $45(16-100)$ & $27(20-88)$ & $52(20-100)$ & $47(16-93)$ & $<0.001$ & $<0.001$ \\
\hline$\geq 30 \%$ & 135 (70\%) & $11(42 \%)$ & 67 (77\%) & $57(72 \%)$ & 0.003 & 0.001 \\
\hline \multicolumn{7}{|l|}{ WBC } \\
\hline Median (range) & $5(0-360)$ & $3(0-15)$ & $5(1-236)$ & $7(1-360)$ & 0.13 & $<0.001$ \\
\hline$\geq 15 \times 10^{9} / 1$ & $65(31 \%)$ & $0(0 \%)$ & $31(34 \%)$ & $34(35 \%)$ & 0.002 & $<0.001$ \\
\hline \multicolumn{7}{|l|}{$\mathrm{LDH}$} \\
\hline Median (range) & $324(116-4835)$ & $259(136-1133)$ & $340(134-2664)$ & 332 (116-4835) & 0.15 & 0.092 \\
\hline$>600 \mathrm{U} / \mathrm{l}$ & $49(23 \%)$ & $2(8 \%)$ & $21(23 \%)$ & $26(27 \%)$ & 0.13 & 0.15 \\
\hline Cytogenetic risk & & & & & 0.003 & 0.48 \\
\hline Favourable & $8(4 \%)$ & $0(0 \%)$ & $4(4 \%)$ & $4(4 \%)$ & & \\
\hline Intermediate & $135(63 \%)$ & $18(69 \%)$ & $62(69 \%)$ & $55(57 \%)$ & & \\
\hline Unfavourable & 47 (22\%) & $8(31 \%)$ & $21(23 \%)$ & $18(19 \%)$ & & \\
\hline Not available & $23(11 \%)$ & $0(0 \%)$ & $3(3 \%)$ & $20(21 \%)$ & & \\
\hline Molecular markers & & & & & 0.27 & 0.45 \\
\hline NPMC+/ITD- & $13(7 \%)$ & $1(4 \%)$ & $9(11 \%)$ & $3(4 \%)$ & & \\
\hline Others & $163(93 \%)$ & $23(96 \%)$ & 75 (89\%) & 65 (96\%) & & \\
\hline
\end{tabular}

Patients with promyelocytic leukaemia $(N=14)$ were excluded from this analysis. Abbreviations: BSC, best supportive care only; Aza vs IC, azacitidine versus intensive chemotherapy; $\mathrm{HCT}$, hematopoietic cell transplantation; AML, acute myeloid leukaemia; BM, bone marrow; WBC, white blood cell count; $\mathrm{LDH}$, lactate dehydrogenase; NPMC+/ITD-, cytoplasmic NPM1 without FLT3 internal tandem duplication. Results are reported as $N$ (\%) unless otherwise indicated.

for PR. In the azacitidine group, median time to response from the start of therapy was 4 months (range 3-7 months) and median duration of response was 16 months. Of the 15 azacitidine-treated patients who did not meet criteria for response, 5 patients had a stable disease for 5-15 months.
In the intensive chemotherapy group, $\mathrm{CR}$ was achieved in 46 (51\%) patients after the first induction cycle and, cumulatively, in $58(64 \%)$ patients after the second induction cycle. Median duration of response in the intensive chemotherapy group was 11 months. 
Table 2 Treatment outcome of patients treated with azacitidine or intensive chemotherapy

\begin{tabular}{|c|c|c|c|c|c|}
\hline & \multirow{2}{*}{$\begin{array}{l}\text { Azacitidine } \\
\quad(N=26)\end{array}$} & \multicolumn{2}{|c|}{ Intensive chemotherapy $(N=90)$} & \multicolumn{2}{|r|}{$P$-value } \\
\hline & & All $(N=90)$ & Excl. allo-SCT $(N=76)$ & Aza vs all IC & Aza vs IC excl. allo-SCT \\
\hline \multicolumn{6}{|l|}{ Overall survival } \\
\hline 1-year & $57 \%$ & $56 \%$ & $50 \%$ & $0.93^{1}$ & $0.80^{1}$ \\
\hline 2-year & $35 \%$ & $35 \%$ & $31 \%$ & $0.92^{1}$ & $0.50^{1}$ \\
\hline Response, overall & $11(42 \%)$ & $68(76 \%)$ & $54(71 \%)$ & $<0.001$ & 0.005 \\
\hline$C R$ & $9(35 \%)$ & $63(70 \%)$ & $49(65 \%)$ & & \\
\hline PR & $2(8 \%)$ & $5(6 \%)$ & $5(7 \%)$ & & \\
\hline No $C R$ or $P R$ & $15(58 \%)$ & $22(24 \%)$ & $22(29 \%)$ & & \\
\hline \multicolumn{6}{|l|}{ Early death } \\
\hline within 4 weeks & $1(4 \%)$ & $4(4 \%)$ & $4(4 \%)$ & $0.88^{1}$ & $0.79^{1}$ \\
\hline within 8 weeks & $2(8 \%)$ & $11(12 \%)$ & $11(12 \%)$ & $0.51^{1}$ & $0.40^{1}$ \\
\hline \multicolumn{6}{|c|}{ Relapse/death after response } \\
\hline within 1 year & $4(36 \%)$ & $39(57 \%)$ & $34(63 \%)$ & $0.21^{1}$ & $0.18^{1}$ \\
\hline within 2 years & $5(45 \%)$ & $42(62 \%)$ & $37(69 \%)$ & $0.30^{1}$ & $0.14^{1}$ \\
\hline \multicolumn{6}{|c|}{ Days in hospital, median (range) } \\
\hline month $1-3$ & $0.5(0-30)$ & $56(2-85)$ & $54(2-85)$ & $<0.001$ & 0.029 \\
\hline month 4-6 & $0(0-8)$ & $0(0-81)$ & $0(0-81)$ & 0.036 & 0.006 \\
\hline \multicolumn{6}{|c|}{ RBC transfusions, median per month (range) } \\
\hline month $1-3$ & $2.7(0-10)$ & $7(0-32)$ & $7(0-32)$ & $<0.001$ & $<0.001$ \\
\hline month 4-6 & $0(0-13)$ & $1(0-8)$ & $0(0-8)$ & 0.97 & 0.65 \\
\hline \multicolumn{6}{|c|}{ PLT transfusions, median per month (range) } \\
\hline month $1-3$ & $0.3(0-7)$ & $5(0-19)$ & $5(0-19)$ & $<0.001$ & $<0.001$ \\
\hline month 4-6 & $0(0-1)$ & $0(0-8)$ & $0(0-8)$ & 0.016 & 0.047 \\
\hline
\end{tabular}

${ }^{1}$ Log rank test. Patients with promyelocytic leukaemia $(N=14)$ were excluded from this analysis. Abbreviations: excl. allo-SCT, excluding patients undergoing allogeneic hematopoietic stem cell transplantation; IC, intensive chemotherapy; Aza, azacitidine; vs, versus; Cl, confidence interval; $\mathrm{CR}$, complete remission; $\mathrm{PR}$, partial remission; RBC, red blood cell; PLT, platelet. Results are reported as $N(\%)$ unless otherwise indicated.

\section{Early mortality and supportive care}

The 4- and 8-week mortality rates and the relapse rates were not significantly different in the azacitidine group compared with the intensive chemotherapy group. However, the number of days in the hospital was significantly lower in patients treated with azacitidine compared to intensive chemotherapy during the first three months $(0.5$ versus 56 days, $P<0.001)$ and the following 3 months, i.e. months $4-6$ (0 (range $0-8$ ) versus 0 (range $0-81$ ) days, $P=0.036$ ) after diagnosis (Table 2; Additional file 3: Figure S2A). Patients treated with azacitidine needed less red blood cell transfusions (2.7 versus $7, P<0.001$ ) and less platelet transfusions (0.3 versus $5, P<0.001)$ during the first three months after diagnosis compared to patients treated with intensive chemotherapy, but the number of red blood cell transfusions during months 4-6 was similar in both treatment groups ( 0 versus $0.7, P=0.97$ ) (Table 2; Additional file 3: Figure S2 B, C). Similar results were obtained when excluding patients who underwent allo-SCT (Table 2).

\section{Complications and causes of death}

To compare the number of complications and the causes of death, we selected patients who were treated in the time period that azacitidine was used (2009-2012). Grade 3/4 infections occurred in 9 (35\%) azacitidinetreated patients, in 9 (32\%) BSC-treated patients, and in all 46 (98\%, 1 missing) patients who received intensive chemotherapy. Of the patients treated with azacitidine, 17 (65\%) had grade $3 / 4$ anaemia or thrombocytopenia at some time during the treatment. Causes of death in patients treated with azacitidine, BSC, or intensive chemotherapy were disease progression in 8 (62\%), 19 (79\%), and $12(50 \%)$ patients, respectively; infection in combination with progressive disease in 4 (31\%), 5 (21\%), and 4 (17\%) patients, respectively; and infection without disease progression in $0(0 \%)$ azacitdine and BSC patients, but in 7 (30\%) intensive chemotherapy patients. One patient treated with intensive chemotherapy died because of ischemic heart disease. The patient who was treated with azacitidine and allo-SCT died because of graftversus-host disease. 


\section{Impact of patient- and disease related factors on overall survival}

Median OS of all 227 patients was 7.8 months. Patients with a good performance score $(0-1)$ at baseline had a better OS than patients with an adverse score $(\geq 2)(12.6$ versus 4.0 months, respectively; $P<0.001$ ) (Additional file 4: Figure S3A and Additional file 5: Table S2). Cytogenetic risk significantly predicted the survival with a median OS of 5.9 months in patients with favourablerisk cytogenetics, excluding APL patients, 9.7 months in patients with intermediate risk cytogenetics, and 3.6 months in patients with unfavourable-risk cytogenetics $(P<0.001)$ (Additional file 4: Figure S3B and Additional file 5: Table S2). Patients with a translocation $t(15 ; 17)$ (APL) had a superior OS (median not reached) compared to other AML patients. The median OS of patients with no karyotype available was 1.9 months, which was similar to the OS of patients with unfavourable-risk cytogenetics $(P=0.33)$ (Additional file 4: Figure S3B and Additional file 5: Table S2). The nucleophosmin 1 (NPM1) mutation status and presence of FLT3-internal tandem duplication (ITD) were determined in 67 of the 80 patients with a normal karyotype. Nine patients had cytoplasmic NPM1 without FLT3-ITD (NPMc+/ITD-). Although numbers are small, a trend towards better OS was observed in these patients compared to patients without NPMc+/ITD(median OS 29.5 versus 8.5 months; $P=0.12$; Additional file 4: Figure S3C).

\section{Impact of treatment on overall survival}

The OS in the different treatment groups is depicted in Figure 1. The OS was similar in patients receiving azacitidine and patients receiving intensive chemotherapy (Table 2; 1-year OS 57\% versus 56\%, $P=0.93$; 2-year OS $35 \%$ versus $35 \%, P=0.92$ ). Also when we compared the OS

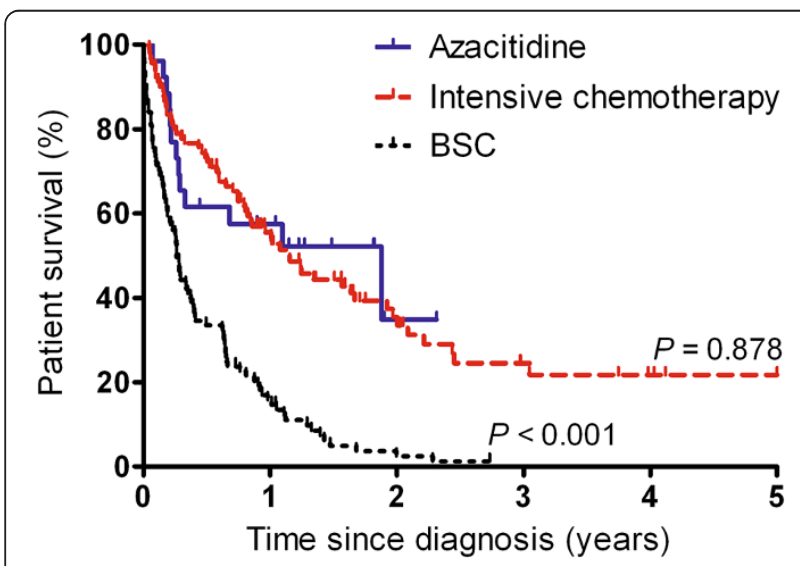

Figure 1 Overall survival by treatment strategy. The OS is similar in patients who were treated with azacitidine $(N=26)$ and intensive chemotherapy (IC; $N=90)$, and is worse in patients who received BSC $(N=97)$. of patients treated since 2009, when azacitidine became available, we observed a similar OS in patients receiving azacitidine and patients receiving intensive chemotherapy (Additional file 6: Figure S4; 1-year OS 57\% versus 51\%, $P=0.80$; 2-year OS $35 \%$ versus $38 \%, P=1.00$ ). Since baseline differences were present among patients treated with azacitidine versus intensive chemotherapy, we assessed the OS in the subgroups of patients aged $\geq 70$ years, patients with HCT comorbidity score $>0$, patients with performance scores $<2$, patients with $<30 \%$ BM blasts, and patients with $<15 \times 10^{9} / 1 \mathrm{WBC}$ 's. Also in these subgroups, no significant differences in OS were observed between azacitidine and intensive chemotherapy $(P=0.74 ; P=0.71$; $P=0.25 ; P=0.71 ; P=0.95$, respectively).

Patients who received BSC had a significantly worse OS (1-year and 2-year OS $16 \%$ and 2\%) compared to azacitidine and intensive chemotherapy $(P<0.001$ and $P<0.001)$. When we selected for patients with a good performance score $(<2)$, which was the lesser part of the BSC group, we still observed a significantly worse OS in the BSC group compared to azacitidine $(P=0.025)$ and intensive chemotherapy $(P=0.004)$.

The median OS of fifteen patients who underwent alloSCT was 22.5 months from the date of diagnosis, while other patients treated with intensive chemotherapy or azacitidine had a median OS of 12.9 months $(\mathrm{p}=0.05)$. After allo-SCT, five patients died due to a relapse $(N=4)$ or graft versus host disease $(N=1)$.

Of the 90 patients treated with intensive chemotherapy, 51 (57\%) were included in a clinical trial and 39 (43\%) were treated off-study. Patients treated off-study had more comorbidities $(\mathrm{p}<0.001)$ than patients included in a trial. Between these patient groups, no differences in overall response rates $(75 \%$ versus $72 \% ; P=0.81)$, and no differences in median OS (14.9 versus 12.9 months, respectively; $P=0.77$ ) were observed (data not shown).

\section{Predictors for overall survival}

To assess whether the OS was similar in patients who were treated with azacitidine and intensive chemotherapy after correction for patient- and disease related factors, we performed a multivariate regression analysis. First, we determined which factors were associated with OS. In univariate analysis, unfavourable OS was associated with BSC (versus azacitidine), unfavourable cytogenetic risk or cytogenetic risk not evaluated (versus intermediate risk), age $\geq 70$ years, performance score $\geq 2$, and LDH $>600 \mathrm{U} / 1$ (Additional file 5: Table S2). Next, we selected from univariate analysis predictors for $\mathrm{OS}$ with $P<0.10$. Multivariate analysis confirmed BSC, unfavourable cytogenetic risk, and $\mathrm{LDH}>600 \mathrm{U} / \mathrm{l}$ as independent adverse predictors for OS. The survival of patients treated with azacitidine versus intensive chemotherapy was not significantly different after correction for these factors $(\mathrm{P}=0.84)$. 


\section{Discussion}

In this single centre retrospective study, treatment results of 227 newly diagnosed consecutive AML patients aged $\geq 60$ years who have been treated with BSC, azacitidine, or intensive chemotherapy, were analysed. This study confirms the dismal prognosis of older AML patients who receive only BSC, which was either related to adverse characteristics at baseline or to the treatment type. To optimise treatment in older patients who are unfit for chemotherapy, new therapies are developed, including azacitidine. A treatment benefit for azacitidine compared to BSC was observed in a post-hoc analysis of the AML patients in the AZA-001 randomized trial [19]. In the same trial, also a limited number of patients treated with intensive chemotherapy was included, but no significant differences in OS were observed between patients treated with azacitidine versus intensive chemotherapy.

In our retrospective study, despite the limitations of the relatively small number of patients and disparities between the treatment groups, we observed no significant differences in OS in patients treated with azacitidine compared to intensive chemotherapy. Also a time-dependent effect could be excluded. When corrected for baseline differences in a multivariate analysis, a HR of 1.07 was found with a 95\% CI of 0.58-2.0 when azacitidine and intensive chemotherapy were compared. Despite relatively small numbers resulting in a wide $\mathrm{CI}$, our point estimate $(\mathrm{HR}=1.07)$ does suggest a comparable treatment effect of azacitidine treatment versus intensive chemotherapy in older AML patients with good performance scores and low WBC counts. Comparable results have been reported recently by the MD Andersen Cancer Centre in a cohort study of 671 patients, including 114 patients treated with hypomethylation-based (either azacitidine or decitabine) therapy [22]. In this study they also reported a significant difference in CR rates but similar OS in patients treated with epigenetic therapy versus intensive chemotherapy. These observations, in a larger cohort, are in line with our observations and might suggest that the currently used response criteria are not sufficient for evaluating some (less intensive) treatment strategies. Further, in the perspective of comparing intensive treatment with less intensive treatment, it is also interesting to note that a small prospective randomised trial between chemotherapy and low-dose cytarabine did not result in a survival benefit for intensive treatment [23].

An important issue, though difficult to analyse, is the reason why some patients received only BSC, others azacitidine and others intensive chemotherapy. The patients receiving azacitidine differed from the intensive chemotherapy patients in terms of older age, and more comorbidities, but also better performance, lower WBC counts, and lower BM blast counts, while the BSC group consisted of older patients with a high cytogenetic risk score, a poor performance score, and a high HCTcomorbidity index. Apparently, although no defined guidelines were used, the treating physicians seem to have integrated these baseline characteristics in their clinical decisions. Azacitidine is currently only registered for the treatment of AML with bone marrow blasts between $20 \%$ and $30 \%$. However, we have recently analysed a cohort of 55 AML patients treated in different hospitals with azacitidine, which included $31 \%$ patients with $\geq 30 \%$ bone marrow blasts. A comparable OS and response rates were demonstrated in patients with $<30 \%$ and $\geq 30 \%$ bone marrow blasts (van der Helm, 2013, in publication). These findings are in line with the results of the Italian named patient program and a German trial [20,21]. An additional advantage of azacitidine is the tolerability $[19,24,25]$, which is reflected in our study by a lower number of days in the hospital and a lower number of red blood cell- and platelet transfusions compared to intensive chemotherapy. In addition, only two of 26 azacitidine-treated patients discontinued treatment because of drug toxicity.

The ongoing phase III trial of azacitidine versus BSC versus intensive chemotherapy (AZA-AML-001 trial) is expected to finally provide the decisive answers for the optimal treatment schedule for elderly AML patients. Recently, the results have been reported of a large phase III trial, comparing the efficacy and safety of decitabine $\left(20 \mathrm{mg} / \mathrm{m}^{2}\right.$, days $\left.1-5\right)(N=242)$ with treatment choice (supportive care $(N=28)$ and low dose cytarabine $(N=$ 215) of older patients with newly diagnosed AML and poor- or intermediate-risk cytogenetics [26]. The authors concluded that there was a significant improvement in median OS with decitabine versus treatment choice.

\section{Conclusions}

Azacitidine treatment is associated with a comparable OS but higher tolerability in a subgroup of older AML patients compared with intensive chemotherapy. Patients receiving BSC had a poor prognosis. Therefore, our data suggest that azacitidine treatment might be a valuable alternative to intensive chemotherapy and should be considered instead of BSC in older AML patients.

\section{Methods}

\section{Patients and data collection}

For this retrospective study, data has been collected from 227 consecutive AML patients of 60 years and older who were diagnosed and treated between January 2002 and May 2012 at the University Medical Centre Groningen. Patients were entered in the study after approval by a scientific review committee and the University Medical Center Groningen Institutional Review Board. Informed consent was obtained in accordance with the Declaration of Helsinki. Data has been collected by studying health 
records of individual patients between October 2011 and October 2012. The minimal follow-up time was six months. Diagnoses were made using French-American-British criteria and World Health Organization (WHO)-2008 criteria $[27,28]$. Cytogenetic risk was defined according to the National Comprehensive Cancer Network (NCCN) guidelines [29]. Baseline comorbidity was quantified by the haematopoietic cell transplantation (HCT) comorbidity index, which was previously demonstrated to be a predictive score in AML patients over 60 years of age treated with intensive chemotherapy $[14,30,31]$.

\section{Treatment}

Azacitidine was available in The Netherlands from December 2008 onwards in a compassionate named patient program. Azacitidine was administered subcutaneously at the approved schedule of $75 \mathrm{mg} / \mathrm{m}^{2} /$ day during 7 days every 28 days. It was intended to give at least 6 cycles of azacitidine and to continue treatment until progression in patients who responded well. Dose reductions and delays of treatment cycles could be made.

Intensive chemotherapy was administered according to one of the HOVON studies [9,32,33], which all contain standard dose cytarabine and an anthracycline (www.hovon.nl). As part of the subsequent HOVON studies, patients were randomised to receive or not G-CSF, intermediate dose cytarabine, bevacizumab, clofarabine, or lenalidomide in addition to the chemotherapy. Of the patients treated according to HOVON studies, 57\% was officially included in a HOVON study. Allogeneic haematopoietic stem cell transplantation could be applied following induction therapy. Patients with acute promyelocytic leukaemia (APL) were treated with ATRA-containing chemotherapy, according to the HOVON 79 study [34].

Best supportive care (BSC) consisted of transfusions, antibiotics, and hospital admissions as needed. 6-Mercaptopurine and hydroxycarbamide could be added to the treatment. Red blood cell- or platelet transfusions were given in agreement with general recommendations: $\mathrm{Hb}<8 \mathrm{~g} / \mathrm{dl}$, or higher in case of comorbidity, and platelets $<20 \times 10^{9} / 1$ or higher in case of bleeding or anticoagulant therapy.

\section{Response criteria and study endpoints}

Response was evaluated after every treatment cycle of intensive chemotherapy and azacitidine by blood count and by bone marrow aspirate if available. Morphologic $\mathrm{CR}$ and partial remission (PR) were defined according to IWG-2003 criteria for AML [35]. Response duration was measured from the date at which marrow evaluation took place in patients achieving $\mathrm{CR}$ or $\mathrm{PR}$, until relapse or death or censoring. OS was measured from the date of diagnosis. Patients who remained alive were censored at the time of the last visit to the hospital.

\section{Statistical analysis}

Differences between groups in patient characteristics and response rates were compared using 2 -sided Fisher's exact tests or chi-square tests for categorical variables and Kruskal-Wallis tests or Wilcoxon tests for quantitative variables, unless otherwise indicated. Survival curves were estimated with the Kaplan-Meier method and differences in survival were calculated by logrank tests. Predictive factors for OS were analysed by Wald tests for univariate and multivariate comparisons. For multivariate analysis, we selected variables with $P<0.10$ in univariate analysis. Cox proportional hazards regression models were used to estimate hazard ratios (HR) and associated 95\% confidence intervals $(\mathrm{CI})$. As we aimed to compare azacitidine treatment with intensive chemotherapy and BSC, the variable "treatment strategy" was pre-added to the multivariate regression model. A $P$-value $<0.05$ was considered significant. SPSS-20 was used for analysis.

\section{Findings}

Response rates were lower in older AML patients treated with azacitidine compared to intensive chemotherapy, however, overall survival in these treatment groups was comparable.

\section{Additional files}

Additional file 1: Figure S1. Flow diagram of the study population. Between January 2002 and May 2012, 227 consecutive AML patients aged $\geq 60$ years were diagnosed and treated in our hospital. Abbreviations: $\mathrm{AML}$, acute myeloid leukaemia; $\mathrm{APL}$, acute promyelocytic leukaemia.

Additional file 2: Table S1. Baseline characteristics of patients who underwent allogeneic haematopoietic stem cell transplantation.

Additional file 3: Figure S2. Supportive care during treatment with azacitidine or intensive chemotherapy. (A) The number of days in the hospital was lower in patients treated with azacitidine compared to intensive chemotherapy during the first three months $(P<0.001)$ and the following 3 months $(P=0.036)$ after diagnosis. (B) Patients treated with azacitidine needed less platelet $(P L T)$ transfusions during the first three months $(P<0.001)$ and the following three months $(P<0.016)$ compared to intensive chemotherapy. (C) Patients treated with azacitidine needed less red blood cell $(\mathrm{RBC})$ transfusions $(P<0.001)$ during the first three months compared to intensive chemotherapy. The median, $5^{\text {th }}, 25^{\text {th }}, 75^{\text {th }}$ and $95^{\text {th }}$ percentile are depicted.

Additional file 4: Figure S3. Impact of patient and disease factors on overall survival. (A) Patients with WHO performance score $0-1$ had a superior OS compared to patients with performance score $\geq 2$. (B) The cytogenetic risk score was a strong predictor for OS. Patients with acute promyelocytic leukaemia (APL) had a favourable survival. The OS of patients with no cytogenetics available was comparable to patients with unfavourable-risk cytogenetics. (C) In patients with a normal karyotype, a trend towards better OS was observed in the presence of cytoplasmic NPM1 without FLT3-ITD (NPMC+/ITD-) compared to other patients.

Additional file 5: Table S2. Predictors for overall survival: univariate and multivariate analysis.

Additional file 6: Figure S4. Overall survival by treatment strategy in the time period that azacitidine was available. The OS was similar in patients who were treated with azacitidine $(N=26)$ and intensive chemotherapy $(I C ; N=47)$, and was worse in patients who received BSC $(N=28)$. 


\section{Abbreviations}

Allo-SCT: Allogeneic haematopoietic stem cell transplantation; AML: Acute myeloid leukaemia; APL: Acute promyelocytic leukaemia; ATRA: All-trans retinoic acid; BM: Bone marrow; BSC: Best supportive care; Cl: Confidence interval; CR: Complete remission; HCT: Haematopoietic cell transplantation; HR: Hazard ratio; ITD: Internal tandem duplication; LDH: Lactate dehydrogenase; NPM1: Nucleophosmin 1; OS: Overall survival; PR: Partia remission; RBC: Red blood cell transfusion; PLT: Platelet transfusion; WBC: White blood cell count; WHO: World Health Organization.

\section{Competing interests}

The authors declare that they have no competing interests.

\section{Authors' contributions}

$\mathrm{LHvdH}$ and ERMS provided the conception and design of the study, acquisition of data, analysis and interpretation of data, drafting the manuscript, revised it critically for important intellectual content. NJGMV supplied the analysis and interpretation of data. SMGJD enrolled patients and provided acquisition of data. ABM and EvdB provided acquisition of data. EV and $\mathrm{GH}$ provided the conception and design of the study, interpretation of data, drafting the manuscript, revised it critically for important intellectual content. All authors read and approved the final manuscript.

\section{Author details}

${ }^{1}$ Department of Haematology, University Medical Centre Groningen, University of Groningen, Hanzeplein 1, Groningen 9713 GZ, The Netherlands. 2Department of Laboratory Medicine, University Medical Centre Groningen, University of Groningen, Groningen, The Netherlands. ${ }^{3}$ Department of Genetics, University Medical Centre Groningen, University of Groningen, Groningen, The Netherlands.

Received: 31 January 2013 Accepted: 5 April 2013

Published: 16 April 2013

\section{References}

1. Estey E, Dohner H: Acute myeloid leukaemia. Lancet 2006, 368(9550): 1894-1907.

2. Pollyea DA, Kohrt HE, Medeiros BC: Acute myeloid leukaemia in the elderly: a review. Br J Haematol 2011, 152(5):524-542.

3. Appelbaum FR, Gundacker H, Head DR, Slovak ML, Willman CL, Godwin JE, et al: Age and acute myeloid leukemia. Blood 2006, 107(9):3481-3485.

4. Estey E: Acute myeloid leukemia and myelodysplastic syndromes in older patients. J Clin Oncol 2007, 25(14):1908-1915.

5. de Jonge HJ, de Bont ES, Valk PJ, Schuringa JJ, Kies M, Woolthuis CM, et al: AML at older age: age-related gene expression profiles reveal a paradoxical down-regulation of p16INK4A mRNA with prognostic significance. Blood 2009, 114(14):2869-2877.

6. Estey EH: General approach to, and perspectives on clinical research in, older patients with newly diagnosed acute myeloid leukemia. Semin Hematol 2006, 43(2):89-95.

7. Krug $U$, Buchner T, Berdel WE, Muller-Tidow C: The treatment of elderly patients with acute myeloid leukemia. Dtsch Arztebl Int 2011, 108(51-52):863-870.

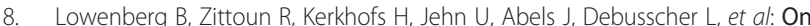
the value of intensive remission-induction chemotherapy in elderly patients of $65+$ years with acute myeloid leukemia: a randomized phase III study of the European Organization for Research and Treatment of Cancer Leukemia Group. J Clin Oncol 1989, 7(9):1268-1274.

9. Lowenberg B, Ossenkoppele GJ, van Putten W, Schouten HC, Graux C, Ferrant $A$, et al: High-dose daunorubicin in older patients with acute myeloid leukemia. N Engl J Med 2009, 361(13):1235-1248.

10. Juliusson G, Antunovic P, Derolf A, Lehmann S, Mollgard L, Stockelberg D, et al: Age and acute myeloid leukemia: real world data on decision to treat and outcomes from the Swedish Acute Leukemia Registry. Blood 2009, 113(18):4179-4187.

11. Burnett AK, Milligan D, Prentice AG, Goldstone AH, McMullin MF, Hills RK, et al: A comparison of low-dose cytarabine and hydroxyurea with or without all-trans retinoic acid for acute myeloid leukemia and high-risk myelodysplastic syndrome in patients not considered fit for intensive treatment. Cancer 2007, 109(6):1114-1124.
12. Eleni LD, Nicholas ZC, Alexandros S: Challenges in treating older patients with acute myeloid leukemia. J Oncol 2010, 2010:943823.

13. Kantarjian H, O'brien S, Cortes J, Giles F, Faderl S, Jabbour E, et al: Results of intensive chemotherapy in 998 patients age 65 years or older with acute myeloid leukemia or high-risk myelodysplastic syndrome: predictive prognostic models for outcome. Cancer 2006, 106(5):1090-1098.

14. Savic A, Kvrgic V, Rajic N, Urosevic I, Kovacevic D, Percic I, et al: The hematopoietic cell transplantation comorbidity index is a predictor of early death and survival in adult acute myeloid leukemia patients. Leuk Res 2012, 36(4):479-482.

15. Krug U, Rollig C, Koschmieder A, Heinecke A, Sauerland MC, Schaich M, et al: Complete remission and early death after intensive chemotherapy in patients aged 60 years or older with acute myeloid leukaemia: a webbased application for prediction of outcomes. Lancet 2010, 376(9757): 2000-2008.

16. Walter RB, Othus M, Borthakur G, Ravandi F, Cortes JE, Pierce SA, et al: Prediction of early death after induction therapy for newly diagnosed acute myeloid leukemia with pretreatment risk scores: a novel paradigm for treatment assignment. J Clin Oncol 2011, 29(33):4417-4423.

17. van der Helm LH, Alhan C, Wijermans PW, van Marwijk KM, Schaafsma R, Biemond BJ, et al: Platelet doubling after the first azacitidine cycle is a promising predictor for response in myelodysplastic syndromes (MDS), chronic myelomonocytic leukaemia (CMML) and acute myeloid leukaemia (AML) patients in the Dutch azacitidine compassionate named patient programme. Br J Haematol 2011, 155(5):599-606.

18. Fenaux P, Mufti GJ, Hellstrom-Lindberg E, Santini V, Finelli C, Giagounidis A, et al: Efficacy of azacitidine compared with that of conventional care regimens in the treatment of higher-risk myelodysplastic syndromes: a randomised, open-label, phase III study. Lancet Oncol 2009, 10(3):223-232.

19. Fenaux P, Mufti GJ, Hellstrom-Lindberg E, Santini V, Gattermann N, Germing U, et al: Azacitidine prolongs overall survival compared with conventional care regimens in elderly patients with low bone marrow blast count acute myeloid leukemia. J Clin Oncol 2010, 28(4):562-569.

20. Maurillo L, Venditti A, Spagnoli A, Gaidano G, Ferrero D, Oliva E, et a: Azacitidine for the treatment of patients with acute myeloid leukemia: report of 82 patients enrolled in an Italian Compassionate Program. Cancer 2012, 118(4):1014-1022.

21. Al-Ali HK, Jaekel $N$, Junghanss $C$, Maschmeyer $G$, Krahl R, Cross M, et al: Azacitidine in patients with acute myeloid leukemia medically unfit for or resistant to chemotherapy: a multicenter phase I/II study. Leuk Lymphoma 2012, 53(1):110-117.

22. Quintas-Cardama A, Ravandi F, Liu-Dumlao T, Brandt M, Faderl S, Pierce S, et al: Epigenetic therapy is associated with similar survival compared with intensive chemotherapy in older patients with newly diagnosed acute myeloid leukemia. Blood 2012, 120(24):4840-4845.

23. Tilly H, Castaigne S, Bordessoule D, Casassus P, Le Prise PY, Tertian G, et al: Low-dose cytarabine versus intensive chemotherapy in the treatment of acute nonlymphocytic leukemia in the elderly. J Clin Oncol 1990, 8(2): 272-279.

24. Seymour JF, Fenaux P, Silverman LR, Mufti GJ, Hellstrom-Lindberg E, Santini V, et al: Effects of azacitidine compared with conventional care regimens in elderly ( $>/=75$ years) patients with higher-risk myelodysplastic syndromes. Crit Rev Oncol Hematol 2010, 76(3):218-227.

25. Santini V, Fenaux P, Mufti GJ, Hellstrom-Lindberg E, Silverman LR, List A, et al: Management and supportive care measures for adverse events in patients with myelodysplastic syndromes treated with azacitidine. Eur J Haematol 2010, 85(2):130-138.

26. Kantarjian HM, Thomas XG, Dmoszynska A, Wierzbowska A, Mazur G, Mayer J et al: Multicenter, randomized, open-label, phase III trial of decitabine versus patient choice, with physician advice, of either supportive care or low-dose cytarabine for the treatment of older patients with newly diagnosed acute myeloid leukemia. J Clin Oncol 2012, 30(21):2670-2677.

27. Bennett JM, Catovsky D, Daniel MT, Flandrin G, Galton DA, Gralnick HR, et al: Proposals for the classification of the myelodysplastic syndromes. $\mathrm{Br} \mathrm{J}$ Haematol 1982, 51(2):189-199.

28. Vardiman JW, Thiele J, Arber DA, Brunning RD, Borowitz MJ, Porwit A, et al: The 2008 revision of the World Health Organization (WHO) classification of myeloid neoplasms and acute leukemia: rationale and important changes. Blood 2009, 114(5):937-951.

29. O'Donnell MR, Abboud CN, Altman J, Appelbaum FR, Arber DA, Attar E, et al Acute myeloid leukemia. J Natl Compr Canc Netw 2012, 10(8):984-1021. 
30. Sorror ML, Maris MB, Storb R, Baron F, Sandmaier BM, Maloney DG, et al: Hematopoietic cell transplantation (HCT)-specific comorbidity index: a new tool for risk assessment before allogeneic HCT. Blood 2005, 106(8): 2912-2919.

31. Giles FJ, Borthakur G, Ravandi F, Faderl S, Verstovsek S, Thomas D, et al: The haematopoietic cell transplantation comorbidity index score is predictive of early death and survival in patients over 60 years of age receiving induction therapy for acute myeloid leukaemia. $\mathrm{Br} J$ Haematol 2007, 136(4):624-627.

32. Lowenberg B, Pabst T, Vellenga E, van Putten W, Schouten HC, Graux C, et al Cytarabine dose for acute myeloid leukemia. N Engl J Med 2011, 364(11): 1027-1036.

33. Ossenkoppele GJ, Stussi G, Maertens J, van Montfort K, Biemond BJ, Breems DM, et al: Addition of bevacizumab to chemotherapy in acute myeloid leukemia at older age: a randomized phase II trial of HOVON/SAKK. Blood 2012, 120(24):4706-4711.

34. Sanz MA, Montesinos P, Rayon C, Holowiecka A, de la Serna J, Milone G, et al: Risk-adapted treatment of acute promyelocytic leukemia based on alltrans retinoic acid and anthracycline with addition of cytarabine in consolidation therapy for high-risk patients: further improvements in treatment outcome. Blood 2010, 115(25):5137-5146.

35. Cheson BD, Bennett JM, Kopecky KJ, Buchner T, Willman CL, Estey EH, et al: Revised recommendations of the International Working Group for Diagnosis, Standardization of Response Criteria, Treatment Outcomes, and Reporting Standards for Therapeutic Trials in Acute Myeloid Leukemia. J Clin Oncol 2003, 21(24):4642-4649.

doi:10.1186/1756-8722-6-29

Cite this article as: van der Helm et al: Azacitidine might be beneficial in a subgroup of older AML patients compared to intensive chemotherapy: a single centre retrospective study of 227 consecutive patients. Journal of Hematology \& Oncology 2013 6:29.

\section{Submit your next manuscript to BioMed Central and take full advantage of:}

- Convenient online submission

- Thorough peer review

- No space constraints or color figure charges

- Immediate publication on acceptance

- Inclusion in PubMed, CAS, Scopus and Google Scholar

- Research which is freely available for redistribution 